\title{
Predicting fatigue crack initiation in fibre metal laminates based on metal fatigue test data
}

\author{
S.W.F. Spronk ${ }^{\mathrm{a}, \mathrm{b}}$, I. Şen ${ }^{\mathrm{a}, *}$, R.C. Alderliesten ${ }^{\mathrm{a}}$ \\ ${ }^{a}$ Structural Integrity $\& 3$ Composites Group, Faculty of Aerospace Engineering, Delft University of Technology, P.O. Box 5058, 2600 GB \\ Delft, The Netherlands \\ ${ }^{b}$ Department of Materials Science and Engineering, Faculty of Engineering and Architecture, Ghent University, \\ Technologiepark-Zwijnaarde 903, B-9052 Zwijnaarde, Belgium
}

\begin{abstract}
A methodology is presented to predict the cycles to crack initiation in a notched fibre metal laminate subjected to cyclic loading. The methodology contains four steps. First, the far-field metal layer stress cycle is obtained using classical laminate theory. Second, the peak stress cycle is estimated from a combination of a handbook solution for the stress concentration factor in a finite isotropic plate, and analytical solutions for the stress concentration for equal situations in infinitely large plates. The third step is to adapt the amplitude of the peak stress cycle to the characteristics of S-N data for monolithic material from the literature to allow for the cycles to initiation to be read from the S-N curve for each metal layer.

In contrast to what can be found hitherto in the literature about predicting the cycles to fatigue crack initiation in fibre metal laminates, the authors of this paper leave no obscurities but rather attempt to bring understanding of the complete path from situation to prediction.

Test results from the literature for Glare 4B-3/2-0.3 have been replicated using the aforementioned methodology. It is shown that it can accurately predict the number of cycles to crack initiation, although the S-N data that is used for the predictions dictates the obtained accuracy. The closer the stress cycle value of the S-N data is to the value of the case analysed, the higher the accuracy obtained. Such a trend was not observed for the stress concentration factor of the S-N curves used, although a choice for S-N data with a different stress concentration factor can cause a significant change in precision. The method is also shown to work for several other fibre metal laminates.
\end{abstract}

Keywords: fibre metal laminates, fatigue, crack initiation, prediction

\section{Introduction}

Fibre metal laminates (FMLs) owe their popularity mainly to their better specific fatigue resistance in comparison to their monolithic aluminium counterparts [1]. Fatigue can be split in two phases. First, the fatigue crack initiation (FCI) phase occurs, which is dominated by material surface conditions owing to the small dimensions of the damage. In the second phase, the damage grows to a larger size and fatigue resistance depends on bulk material parameters rather than the surface [2]. The improved fatigue resistance of FMLs is attributed to its fibre layers which bridge fatigue cracks that appear in the metal layers due to repetitive loading [3]. This work focuses on the crack initiation phase.

If full use is to be made from the fatigue resisting capability of FMLs, it is of vital importance that the amount of load cycles the material is able to survive can be accurately predicted. Although many different methods already exist

\footnotetext{
*Corresponding author. Tel.: +31 (0)15 278 9749;

E-mail address: i.sen@tudelft.nl
}

to describe the fatigue crack growth behaviour of FMLs $[4,5]$, the literature about models that predict FCI in FMLs is rather limited. Currently, a complete description of a method that can predict this number of cycles with a sufficient precision is still lacking. Homan takes the first step towards a prediction model with his proposition to compare the number of cycles to crack initiation for Glare to that for monolithic aluminium by looking at the stress levels in the metal layers rather than the net section stress of the FML [6]. The subsequent use of this stress to get to a prediction of cycles to crack initiation, however, is left untreated. Homan and Schra mention that the presence of residual stress in notched FMLs makes the stress concentration factor (SCF) depend on applied load [7], but no information is given on which SCF should be used to characterise the peak stress cycle in comparison to S-N data. No details are given, moreover, about the selection of one of the many stress ratios that can be computed for an FML, for similar purpose. Chang et al. connect Homan's reasoning to two different models to create an S-N curve for a notched monolithic specimen to allow for a prediction of cycles to crack initiation of FMLs $[8,9]$. Their explana- 
tions, however, do not go further than a rough description of the method by Homan and the connected model, again leaving many implementation details to the reader.

Apart from the incomplete descriptions, the validity of parts of the theories given is questionable as well. Homan sums up layer compliance as if it were stiffness to get to a laminate compliance matrix, does not rotate thermal expansion coefficients using the off-axis matrix, and the given equation for stress concentration along the edge of a circular cut-out does not agree with what is given in the original source. Chang et al. build on Homan's method and provide no alterations.

The current research focuses on creating understanding of the complete methodology to predict the cycles to crack initiation in a notched FML. The methodology implies using Homan's method to find the stress amplitudes in the metal layers at the point of interest, and subsequently comparing these amplitudes to $\mathrm{S}-\mathrm{N}$ data for monolithic aluminium from the literature to get to a prediction of the cycles to crack initiation. In contrast to the works about this topic mentioned above, this paper contains a thorough, directly implementable description of how to predict the number of cycles until a $1[\mathrm{~mm}]$ crack has emerged in FMLs from the layer material properties, dimensions, and loading. Homan's method is, moreover, not bluntly copied from the description provided in [6], but adapted to remove the aforementioned inaccuracies. The body of this work is divided in two parts. First, the method is explained, and second, results of test series are replicated to conclude about its accuracy.

\section{Method}

The suggested methodology to predict FCI in FMLs works in three steps, each of which are explained in this section. First, the far-field metal layer stress is calculated from the applied load using classical laminate theory. Second, the peak stress cycle is estimated, by multiplying a handbook solution for the stress concentration factor in a finite isotropic plate, with the ratio of the analytical solution for the stress concentration for the same case in an infinitely large FML to that in an infinite isotropic plate. Finally, the amplitude of the peak stress cycle is adapted to the characteristics of $\mathrm{S}-\mathrm{N}$ data for monolithic material from the literature using a correction for both the stress cycle and the SCF, after which the number of cycles to initiation is read from the $\mathrm{S}-\mathrm{N}$ curve.

\subsection{Step 1: calculate the far-field metal layer stress}

Classical laminate theory (CLT) is a method to calculate the stresses in each individual layer of a flat, layered material. Many different publications exist that describe the CLT, an example is [10]. New insights caused the authors of this work to make some alterations to the theory as presented in [6], all of which are explained in this section.
Instead of the rule of mixtures that is frequently applied to determine the laminate stiffness from layer properties $[6,8]$, the authors use the ABD-matrix definitions, which allow for a more straightforward inclusion of layers with several orientations into the calculation.

Following Tsai and Hahn [10], the compliance matrix is computed from the inverse of the laminate stiffness matrix rather than adding layer compliance matrices.

The thermal expansion of each layer, moreover, is rotated using the strain rotation matrix, leading to:

$$
\overline{\boldsymbol{\alpha}}=\left\{\begin{array}{l}
\alpha_{1} \cos ^{2}(\theta)+\alpha_{2} \sin ^{2}(\theta) \\
\alpha_{1} \sin ^{2}(\theta)+\alpha_{2} \cos ^{2}(\theta) \\
\left(\alpha_{1}-\alpha_{2}\right) \cos (\theta) \sin (\theta)
\end{array}\right\}
$$

rather than the equation mentioned in [6]. Fictitious thermal line loads and moments $\boldsymbol{N}^{\text {th }}$ and $\boldsymbol{M}^{\text {th }}$ are computed from these thermal strains in the same way for regular strains, see [10]. The total midplane strain $\bar{\varepsilon}_{\text {tot }}^{\circ}$ and curvature $\overline{\boldsymbol{\kappa}}_{\text {tot }}$ can then be calculated using:

$$
\left\{\begin{array}{l}
\bar{\varepsilon}_{\text {tot }}^{\circ} \\
\overline{\boldsymbol{\kappa}}_{\text {tot }}
\end{array}\right\}=\left[\begin{array}{ll}
\boldsymbol{a} & \boldsymbol{b} \\
\boldsymbol{b} & \boldsymbol{d}
\end{array}\right]\left\{\begin{array}{c}
\boldsymbol{N}+\boldsymbol{N}^{\mathrm{th}} \\
\boldsymbol{M}+\boldsymbol{M}^{\mathrm{th}}
\end{array}\right\}
$$

The stress occurring in a layer of the FML as a result of the combination of applied load and thermal expansion can then be calculated in laminate coordinates using:

$$
\boldsymbol{\sigma}=\overline{\boldsymbol{Q}}\left(\bar{\varepsilon}_{\text {tot }}^{\circ}+z \overline{\boldsymbol{\kappa}}_{\text {tot }}-\overline{\boldsymbol{\alpha}} \Delta T\right)
$$

where $\overline{\boldsymbol{Q}}$ is the stiffness matrix of the layer in laminate coordinates, $z$ the distance to the laminate midplane and $\Delta T$ the difference with the temperature at which the laminate is free of thermal stress (i.e. the consolidation temperature). Setting the applied line loads and moments to zero in the equations above results in the thermal stresses in the layers.

The mechanical stress is defined as the stress that would exist in the absence of any thermal effects, and can be calculated by setting the fictitious thermal line loads and moments to zero.

\subsection{Step 2: finding the stress cycle at the notch}

The second step of the FCI prediction methodology is to determine the peak stress cycle in the case analysed, using a stress concentration factor (SCF). No equations are readily available for an SCF in a notched anisotropic plate of finite width. It is assumed all material behaves perfectly elastic. Homan [6] stipulates that the ratio of stress concentration in an infinitely wide FML to that of an infinite isotropic plate is equal or at least close to that same ratio for plates of finite width, or:

$$
\frac{K_{t, \mathrm{FML}}}{K_{t, \text { iso }}}=\frac{K_{t, \mathrm{FML}, \infty}}{K_{t, \text { iso }, \infty}}
$$

where the subscripts iso and $\infty$ stand for isotropic material and infinite width and $K_{t}$ stands for the SCF, which is defined as the ratio of peak stress to nominal stress: $K_{t}=$ $\frac{\sigma_{\text {peak }}}{S_{\text {nom }}}$. 


\begin{tabular}{|c|c|c|c|}
\hline \multicolumn{4}{|c|}{ Notation } \\
\hline \multicolumn{2}{|c|}{ Symbols } & \multicolumn{2}{|c|}{ Subscripts } \\
\hline$a$ & Inverse in-plane laminate stiffness coefficient $[\mathrm{mm} / \mathrm{N}]$ & 1 & The quantity is in the local 1-direction \\
\hline$b$ & Inverse bending-stretching coupling coefficient $[1 / \mathrm{N}]$ & 2 & The quantity is in the local 2-direction \\
\hline$b$ & Inverse bending-stretching coupling coefficient $[1 / \mathrm{N}]$ & $\infty$ & The quantity belongs to an infinitely wide plate \\
\hline$d$ & Inverse bending stiffness coefficient $[1 /(\mathrm{N} \cdot \mathrm{mm})]$ & $a$ & Amplitude \\
\hline$D$ & Hole diameter $[\mathrm{mm}]$ & $\mathrm{ff}$ & Far-field or applied value \\
\hline$f$ & SCF correction factor, see equation (22) [-] & FML & The quantity belongs to a fibre metal laminate \\
\hline$E$ & Young's modulus [MPa] & $\mathrm{i}$ & The quantity describes cycles to crack initiation \\
\hline$G$ & Shear modulus [MPa] & iso & The quantity belongs to isotropic material \\
\hline$K_{t}$ & Stress concentration factor $[-]$ & $k$ & The quantity belongs to layer $k$ \\
\hline$M$ & Line moment on the laminate edge $[\mathrm{N}]$ & $m$ & Mean value \\
\hline$N$ & Line load on the laminate edge $[\mathrm{N} / \mathrm{mm}]$ & net & Net section value, i.e. average over the cross-section \\
\hline$N$ & Number of cycles [cycles] & nom & Nominal value, i.e. not accounting for local effects \\
\hline$Q$ & Plane stress stiffness matrix $[\mathrm{MPa}]$ & peak & Peak or concentrated value \\
\hline$R$ & Stress ratio $[-]$ & $\mathrm{S}-\mathrm{N}$ & The quantity belongs to $\mathrm{S}-\mathrm{N}$ data \\
\hline$S$ & Global direct stress $[\mathrm{MPa}]$ & tot & Total value, i.e. mechanical plus thermal \\
\hline$S_{N}$ & Fatigue strength at fatigue life $N[\mathrm{MPa}]$ & $u$ & Ultimate value \\
\hline$W$ & Plate width $[\mathrm{mm}]$ & $x$ & The quantity is in the global $x$-direction \\
\hline$z$ & $z$-coordinate, starting from the midplane $[\mathrm{mm}]$ & $y$ & The quantity is in the global $y$-direction \\
\hline$\alpha$ & Coefficient of thermal expansion $\left[1 /{ }^{\circ} \mathrm{C}\right]$ & $\theta$ & The quantity is in circumferential direction \\
\hline$\Delta T$ & Temperature difference between curing and testing $\left[{ }^{\circ} \mathrm{C}\right]$ & \multirow{2}{*}{\multicolumn{2}{|c|}{ Superscripts }} \\
\hline$\varepsilon$ & Direct strain $[-]$ & & \\
\hline$\theta$ & Angle between layer 1 -axis and laminate $x$-axis [deg] & & \\
\hline$\kappa$ & Curvature $[1 / \mathrm{mm}]$ & I & The quantity is in the load axis system \\
\hline$\nu$ & Poisson's ratio [-] & $\circ$ & The quantity is the laminate midplane value \\
\hline$\sigma$ & Local direct stress $[\mathrm{MPa}]$ & $\max$ & The value at maximum applied load \\
\hline$\varphi$ & Off-axis loading angle $[\mathrm{deg}]$ & mech & The quantity results from the applied load only \\
\hline$\psi$ & Cylindrical coordinate along cut-out perimeter [deg] & $\min$ & The value at minimum applied load \\
\hline & & th & The quantity results from thermal effects \\
\hline
\end{tabular}

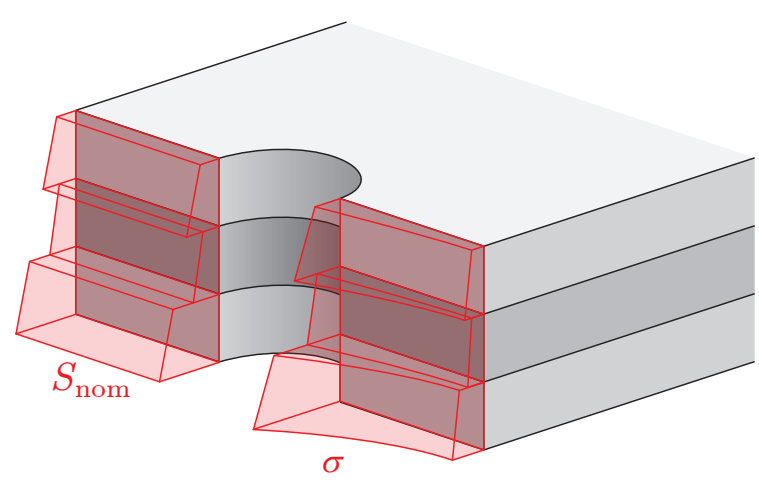

Figure 1. Definition of nominal stress, $S_{\text {nom }}$, and local stress, $\sigma$, in a tensionally loaded notched layered part in plane stress. $S_{\text {nom }}$ is the average value of the $\sigma$ distribution on that $z$-coordinate.

\subsubsection{Nominal stress and local stress}

Two different types of direct stress are used in this paper: a nominal stress $S_{\text {nom }}$ and a local stress $\sigma$. The differences in the definitions of these two types of stresses can be seen in figure 1 . The nominal stress includes the effects of an increase due to a reduction in cross-section, the linear variation due to an applied moment, and the layer-by-layer variation due to differing stiffness values for different layers. The local stress includes the concentrating effect of a cut-out on top of the aforementioned effects.

It is worth mentioning that test data is usually described as a function of net section stress $S_{\text {net }}$. This is the average stress over all layers of the complete laminate cross-section perpendicular to the loading at the peak stress point.

\subsubsection{Stress concentration in a unidirectional stress field}

The tangential stress around the perimeter of a circular cut-out in an infinitely large orthotropic plate due to a unidirectional stress field has been solved by Lekhnitskii [11]. His solution depends on the engineering constants of the laminate, on the off-axis angle and on the cylindrical coordinate along the perimeter of the cut-out. The angular variables are defined here as $\varphi$ and $\psi$, respectively, see also figure 2 .

With all the material and situational data entered in Lehknitskii's equation, the result still depends on the cylindrical coordinate $\psi$. This report focuses on predicting the moment of fatigue crack initiation and thus the highest circumferential stress is of significance.

When a stress field is aligned with the laminate $x$-axis and the laminate consists only of layers with a $0^{\circ}$ or $90^{\circ}$ orientation, the peak stress occurs at the intersections of circular cut-out with the $y$-axis, or $\psi= \pm 90^{\circ}$. In that case, Lehknitskii's equation can be simplified to:

$$
\left.\frac{\sigma_{\theta}}{S}\right|_{\varphi=0^{\circ}, \psi=90^{\circ}}=1+\sqrt{2\left(\sqrt{\frac{E_{x}}{E_{y}}}-\nu_{x y}\right)+\frac{E_{x}}{G_{x y}}}
$$

The tangential stress at the perimeter of a circular hole in an infinitely large isotropic plate can also be calculated 


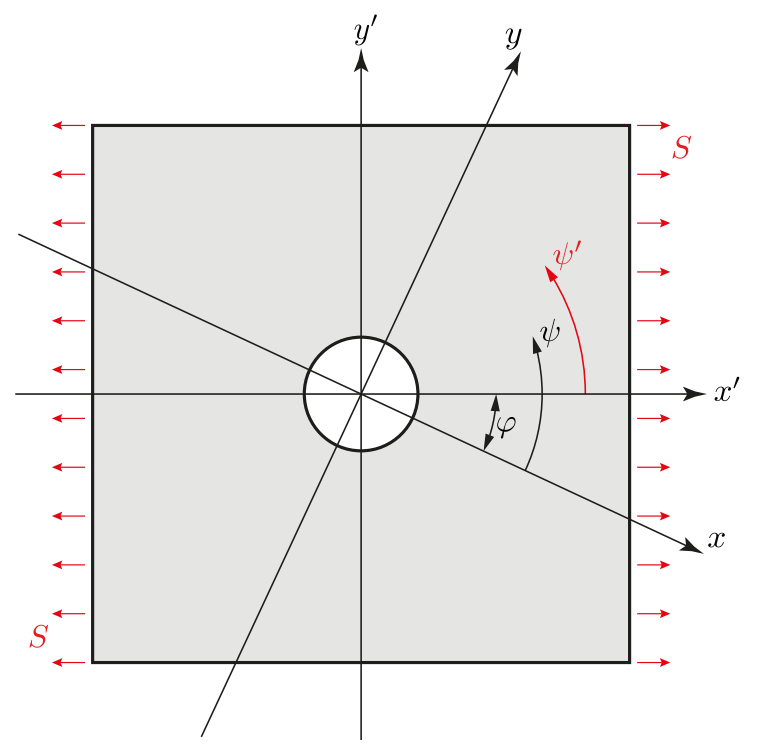

Figure 2. Angle definitions for loading by an off-axis stress field $S$, $x$ and $y$ are the longitudinal and lateral coordinates of the panel, $x^{\prime}$ and $y^{\prime}$ of the stress system

using equation (5), in which case:

$$
\left.\frac{\sigma_{\theta, \text { iso }}}{S}\right|_{\varphi=0^{\circ}, \psi=90^{\circ}}=3
$$

so the SCF for an isotropic plate $K_{t}$, iso, $\infty=3$.

An example of a formula for the maximum SCF for a circular hole in a finite isotropic plate under tension is given by Peterson [12], which also occurs at $\psi=90^{\circ}$ :

$$
\begin{aligned}
K_{t, \text { iso }}= & 2+0.284\left(1-\frac{D}{W}\right)-0.600\left(1-\frac{D}{W}\right)^{2} \\
& +1.32\left(1-\frac{D}{W}\right)^{3}
\end{aligned}
$$

where $D$ is the hole diameter and $W$ the plate width.

Inserting equation (5), equation (7), and $K_{t, \text { iso, } \infty}=3$ into equation (4) results in an expression for the stress concentration in an FML with balanced and symmetric layup of finite width, which is loaded in the direction of the laminate $x$-axis and only contains layers in $0^{\circ}$ or $90^{\circ}$ direction.

There are many different equations available for a variety of stress concentrating effects. Even though equation (7) is used here, it does not mean that the reasoning is limited to this specific case. Different equations for the SCF of a circular cut-out in an isotropic plate of finite width can be used instead of equation (7), e.g. from the ESDU data sheets [13] or Heywood [14]. Alternatively, a completely different set of equations can be used to calculate stress concentrations in cases where the notch has a shape that is different from circular.

\subsubsection{Peak stress not at $\psi= \pm 90^{\circ}$}

For laminates with layer orientation angles other than $\theta=0^{\circ}$ or $90^{\circ}$, or laminates under off-axis loading, the peak

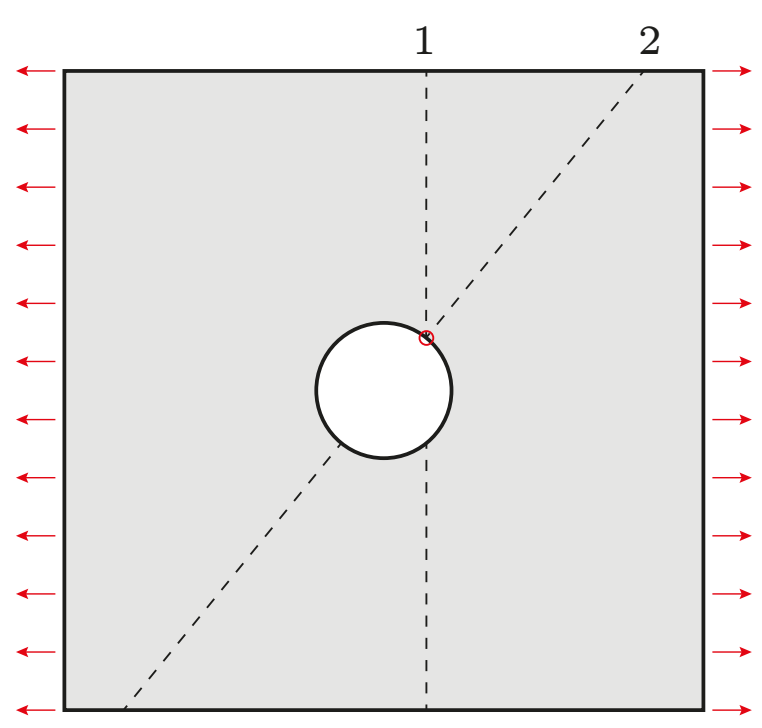

Figure 3. Net section options in case the maximum SCF occurs at $\psi \neq 90^{\circ}$, e.g. at the location indicated

stress due to a load in the $x$-direction can occur at a value of $\psi$ other than $90^{\circ}$. This can be determined using the full solution of stress around a circular cut-out by Lekhnitskii [11]. In this case, two difficulties arise, as detailed below.

First, the net section that is part of the definition of nominal stress becomes difficult to define. Two clear options exist for the choice of the net section in case $\psi \neq 90^{\circ}$, see also figure 3 :

1. the area through the peak stress point and perpendicular to the applied load, or

2. the area perpendicular to the cut-out edge at the peak stress point.

The peak stress acts in circumferential direction at the cut-out edge. Option 2 would thus be correct close to the peak stress point, because the net section is supposed to be perpendicular to the direction of stress. Further away from the hole, however, the direction of stress will be as is applied, and option 1 would thus be more appropriate in this location. The correct net section lies in between the two options, but is hard, if not impossible, to determine. The portion of stress in the plate that flows in a direction other than applied is expected to be relatively small. The net section, moreover, is expected to be smaller than the section without any cut-out. These two arguments lead to the choice of option 1 for the net section.

The second difficulty which arises when the peak stress point lies at a location other than $\psi=90^{\circ}$ is that handbook solutions for stress concentrations in monolithic plates of finite width only specify the maximum SCF rather than the distribution around the cut-out. The SCF in a monolithic plate is thus not known at the peak stress point of its anisotropic equivalent. Two solutions can be applied. The first option is to still use the monolithic SCFs at $\psi=90^{\circ}$ from a handbook for equation (4), and thus assuming that 
the ratio of SCFs between an infinite and finite monolithic plate at $\psi=90^{\circ}$ equals that of the anisotropic material at the peak stress point. Another option is to determine the concentration in the finite width monolithic material at the location necessary using a finite-element analysis, and using again Lekhnitskii for the infinite case.

The method of finding $K_{t}$, FML can thus still be applied when the peak stress occurs at $\psi \neq 90^{\circ}$, although its accuracy might be lower compared to the case with the peak stress point at $\psi=90^{\circ}$.

\subsubsection{Stress concentration in a biaxial stress field}

When biaxial loads are synchronously applied on a laminate, their influence on the tangential stress around the circular cut-out can be summed up, provided the material response remains linear-elastic. When the loads are also applied in the direction of both global laminate axes, the tangential stress at $\psi=0^{\circ}$ or $180^{\circ}$ on the edge of the cut-out in an infinitely large plate becomes:

$$
\begin{aligned}
\sigma_{\theta}= & S_{x}\left(1+\sqrt{2\left(\sqrt{\frac{E_{x}}{E_{y}}}-\nu_{x y}\right)+\frac{E_{x}}{G_{x y}}}\right) \\
& +S_{y}\left(-\sqrt{\frac{E_{x}}{E_{y}}}\right)
\end{aligned}
$$

and the tangential stress at $\psi=90^{\circ}$ or $270^{\circ}$ :

$$
\begin{aligned}
\sigma_{\theta}= & S_{y}\left(1+\sqrt{2\left(\sqrt{\frac{E_{y}}{E_{x}}}-\nu_{y x}\right)+\frac{E_{y}}{G_{x y}}}\right) \\
& +S_{x}\left(-\sqrt{\frac{E_{y}}{E_{x}}}\right)
\end{aligned}
$$

Care should be taken that when loads $S_{x}$ and $S_{y}$ approach each other in magnitude, the severest stress does not necessarily occur at $\psi=0^{\circ}$ or $90^{\circ}$, but could be somewhere in between. In this case the considerations described in section 2.2.3 apply.

\subsubsection{Other cases}

Many other cases exist than those covered by the sections above.

When the loads in a multi-axial load system are not synchronised, a solution for the stress cycle at each point around the notch becomes necessary. This can be obtained algebraically or numerically using the full solution of stress around a circular notch, multiplied with the applied stress as amplitude of a harmonic load, and summing up the effects of each load around the cut-out while making sure the cylindrical coordinate is adapted for each load according the off-axis angle, see also figure 2. Next, of all the load cycles occurring on the cut-out edge, the most severe can be taken to assess the fatigue performance.

If the frequencies of the loads in a multi-axial load system are unequal, the stresses around the notch cease to be of constant amplitude. For fatigue life assessments as a result of variable-amplitude stress cycles, either a equivalent constant amplitude load can be determined that has the same expected damage growth as one repeating set of variable-amplitude cycles, or the sum can be taken of the damage increase for each occurring stress amplitude $[2,15]$. It should be noted that in the latter case, large errors might be produced due to the fact that the damage growth belonging to a stress cycle can be highly dependent on the preceding cycles [2].

If the laminate is unbalanced and/or asymmetric and thus not orthotropic, Lekhnitskii's solution is no longer valid, and a new solution is needed for the SCF in an infinite plate due to uni-axial loading. The circumferential stress around a hole could be found by applying a detailed finite-element analysis of the anisotropic plate of finite width, thereby finding directly the required peak stress and avoiding the need for equation (4) to estimate its value.

\subsubsection{The occurring stress cycle at the notch}

The existence of a thermal stress field in the laminate makes the calculation of the peak stress and stress ratio less straightforward as when only mechanical loads are taken into consideration.

When a notch is made in a flat planar part that is stacked in $z$-direction, the thermal stress field that might be present does not change in the rest of the part if the shape of the notch does not vary in $z$-direction. This effect is illustrated in figure 4. All the parts in the figure are considered to be at room temperature and interlaminar shear stress is neglected, because it is assumed not to play a significant role in FCI. A thermal stress field exists in the cured condition, see figure 4.b, when the individual layers have different CTEs and thus would strain differently if they were not included in a laminate, see figure 4.a. When a vertical cut is made to remove a part of the cured material, see figure 4.c, the thermal stress field that exists in the removed part remains within this piece. There is no in-plane interaction of the thermal stress field with the surrounding material for a part stacked in $z$-direction, because, even though the resulting stress is purely in-plane, the thermal load transfer in this case is only in the stacking direction. A stress concentration effect thus only occurs in the stress field which results from applied load, see figure 4.d, because this stress has to 'flow' around the notch.

The peak stress $\sigma_{k \text {, peak }}$ in each layer $k$ is calculated by multiplying only the nominal mechanical stress $S_{k, \text { nom }}^{\text {mech }}$ occurring in the layer by the SCF and then adding the residual thermal stress $S_{k}^{\text {th }}$ :

$$
\sigma_{k, \text { peak }}=K_{t, \mathrm{FML}} \cdot S_{k, \text { nom }}^{\text {mech }}+S_{k}^{\text {th }}
$$

where the nominal mechanical stress is calculated by only elevating the far-field mechanical stress as a result of the reduced cross-section:

$$
S_{k, \text { nom }}^{\text {mech }}=\left(S_{k, \text { ff }}-S_{k}^{\text {th }}\right)\left(\frac{W}{W-D}\right)
$$




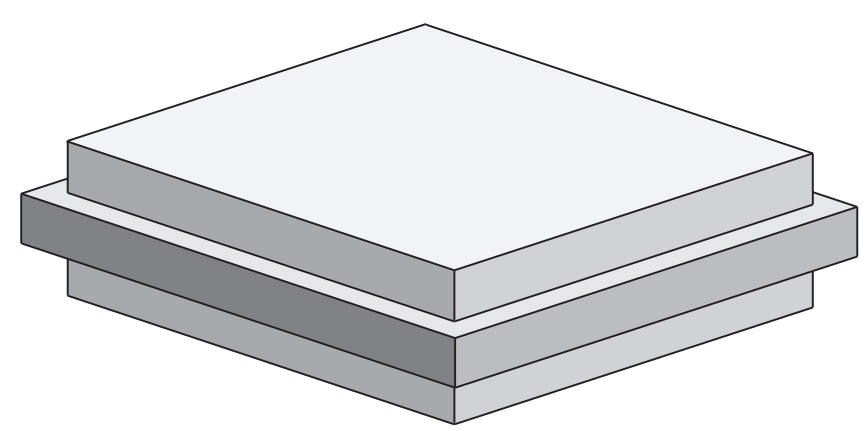

a. The layers have unequal sizes if they are unrestricted

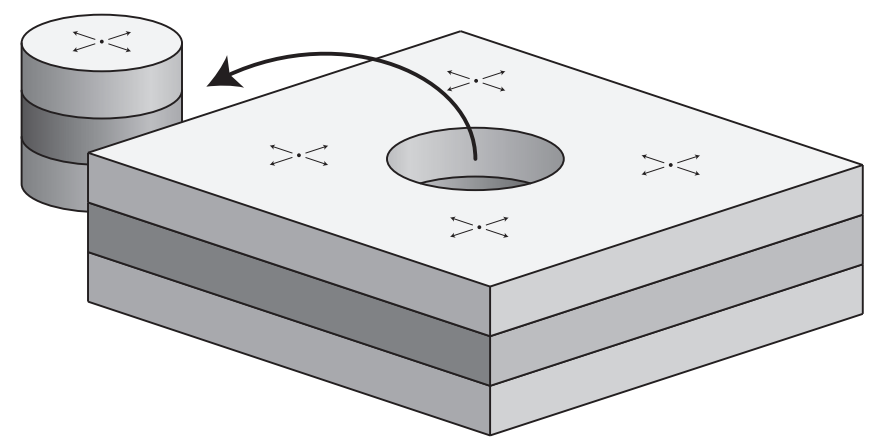

c. Removed material takes its residual stress field with it

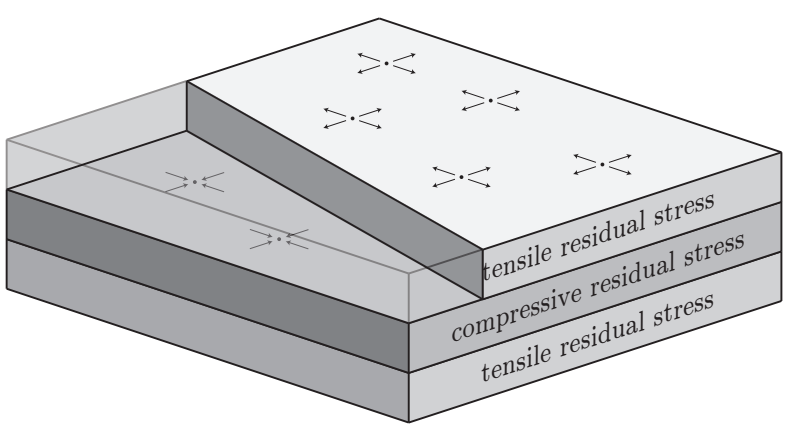

b. Residual stress is present in the cured condition

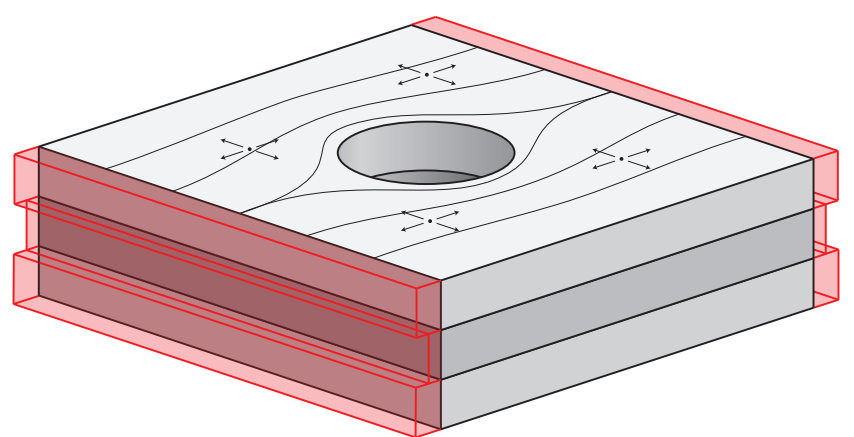

d. Mechanical stress flows around the notch and the SCF applies

Figure 4. A laminate in different conditions

where $S_{k, \text { ff }}$ is the far-field stress in layer $k$ calculated using CLT.

The stress concentrating effect in each layer is no longer independent of the applied load due to the presence of residual stress, and the SCF thus ceases to be a purely geometrical factor. Its value needs to be calculated using the occurring peak and nominal stress values in each layer:

$$
K_{t, k}=\frac{\sigma_{k, \text { peak }}}{S_{k, \text { nom }}}
$$

where the total nominal stress in a layer is the sum of the nominal mechanical stress and the residual thermal stress:

$$
S_{k, \text { nom }}=S_{k, \text { nom }}^{\text {mech }}+S_{k}^{\text {th }}
$$

and $\sigma_{k \text {, peak }}$ is calculated using equation (10).

The value of the SCF which occurs in a layer thus varies between $K_{t, k}^{\min }$, its value at the minimum load of the applied load cycle, and $K_{t, k}^{\max }$, its value at the maximum load of the applied load cycle.

The occurring stress ratio at the point of interest is also different from the stress ratio which is applied in the far-field, and is therefore recalculated as well:

$$
R_{\text {peak }}=\frac{\sigma_{k, \text { peak }}^{\min }}{\sigma_{k, \text { peak }}^{\text {max }}}
$$

\subsection{Step 3: predicting the cycles to crack initiation}

Once the occurring stress cycle has been determined, the fatigue initiation life can be estimated by looking up a calculated stress amplitude in S-N data available in the literature. It has been found that the cycles to failure of notched monolithic aluminium specimens is close to the cycles to a crack length of 1 [mm] in the aluminium layers of an FML with the same shape $[7,16]$. Crack initiation, therefore, is assumed to have taken place at this crack length, and S-N data for monolithic aluminium will be treated as $\mathrm{S}^{-\mathrm{N}_{i}}$ data for the metal layers in a FML, and no factor is used to account for a possible discrepancy between the two.

The question remains which stress to compare to the S-N curve. The stress ratio of this stress and SCF in the analysed case, moreover, should be equal to the stress ratio and $\mathrm{SCF}$ of the $\mathrm{S}-\mathrm{N}$ data for a correct estimation. In case they are not, correction formulae need to be applied to correct for the difference.

\subsubsection{Choice of reference stress}

A reference stress needs to be chosen which is used to compare the analysed case with $\mathrm{S}-\mathrm{N}$ data. Recall from the previous section that the existence of thermal stress results in a variation of the SCF and stress ratio with applied load.

FCI is a material surface phenomenon [2], and it is thus concluded that the stress cycle at the peak stress point is dominating the number of cycles to initiation. It would be ideal to compare the peak stress cycle with data that also relates the peak stress in notched specimens to cycles to failure. S-N data, however, is usually given with nominal stress on the ordinate and number of cycles to failure on the abscissa. 
It is not correct to translate S-N curves based on nominal stress amplitude by simply multiplying with the corresponding SCF to relate peak stress amplitude to cycles to failure. The influence of the SCF, namely, differs for low- and high-cycle fatigue [2].

The combination of nominal stress amplitude, $S_{a, n o m}$, and peak stress ratio, $R_{\text {peak }}$, are therefore used as the reference stress cycle. This allows for a comparison on the basis of nominal stress, while using the correct stress ratio occurring at the point of interest.

\subsubsection{Adapting the load cycle to match the mean stress}

The reference stress cycle has to be converted towards the mean stress level that was used to produce the S-N data with which the amplitude of this stress cycle is to be compared.

The mean value and the amplitude of the reference stress occurring in a layer can be calculated using:

$$
\begin{gathered}
S_{m, \mathrm{nom}}=\left(\frac{1+R_{\mathrm{nom}}}{2}\right) S_{k, \mathrm{nom}}^{\max } \\
S_{a, \mathrm{nom}}=\left(\frac{1-R_{\mathrm{nom}}}{2}\right) S_{k, \mathrm{nom}}^{\max }
\end{gathered}
$$

The amplitude for zero mean reference stress is calculated using the Goodman relation instead of alternative relations, because it is generally a conservative approximation [2]:

$$
\left.S_{a, \mathrm{nom}}\right|_{S_{m}=0}=\frac{S_{a, \mathrm{nom}}}{1+\frac{S_{m, \mathrm{nom}}}{S_{u}}}
$$

To find the amplitude of the stress cycle in the $\mathrm{S}-\mathrm{N}$ curve, $S_{a, \mathrm{~S}-\mathrm{N}}$, that is equivalent to the stress cycle of the test case, the Goodman relation is used once again, this time with the stress ratio of the $\mathrm{S}-\mathrm{N}$ curve, $R_{\mathrm{S}-\mathrm{N}}$ :

$$
S_{a, \mathrm{~S}-\mathrm{N}}=\left.S_{a, \mathrm{nom}}\right|_{S_{m}=0}\left(1-\frac{S_{m, \mathrm{~S}-\mathrm{N}}}{S_{u}}\right)
$$

where the same amplitude for zero mean stress as in equation (17) is used, because it characterises the stress cycle under consideration.

The mean value of the equivalent stress cycle in the S-N data, $S_{m, \text { S-N }}$, is still an unknown at this point. The mean value of any constant-amplitude load cycle can be written as a function of the corresponding amplitude with use of the stress ratio:

$$
S_{\max }=\left(\frac{2}{1-R}\right) S_{a}
$$

in which the denominator will never be zero because $R=1$ would mean there is no load variation. Hence, for the mean value of the peak stress of a load cycle described by the $\mathrm{S}-\mathrm{N}$ curve, it follows that:

$$
\begin{aligned}
S_{m, \mathrm{~S}-\mathrm{N}} & =S_{\max , \mathrm{S}-\mathrm{N}}-S_{a, \mathrm{~S}-\mathrm{N}} \\
& =\left(\frac{2}{1-R_{\mathrm{S}-\mathrm{N}}}-1\right) S_{a, \mathrm{~S}-\mathrm{N}}
\end{aligned}
$$

Equation 18 can now be rewritten using equation 20 to obtain a relation for $S_{a, \mathrm{~S}-\mathrm{N}}$ that is solely dependent on terms that are known and gives the peak stress amplitude in the $\mathrm{S}-\mathrm{N}$ data that is equivalent to the reference stress

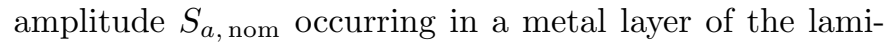
nate under consideration:

$$
\begin{aligned}
S_{a, \mathrm{~S}-\mathrm{N}} & =\left.S_{a, \mathrm{nom}}\right|_{S_{m}=0}\left(1-\frac{\left(\frac{2}{1-R_{\mathrm{S}-\mathrm{N}}}-1\right) S_{a, \mathrm{~S}-\mathrm{N}}}{S_{u}}\right) \\
& =\frac{\left.S_{a, \mathrm{nom}}\right|_{S_{m}=0}}{1+\left.S_{a, \mathrm{nom}}\right|_{S_{m}=0}\left(\frac{\frac{2}{1-R_{\mathrm{S}-\mathrm{N}}}-1}{S_{u}}\right)}
\end{aligned}
$$

\subsubsection{Adapting the load cycle to match the SCF}

A different stress distribution in a plate loaded by a repetitive load leads to a different fatigue life, even if the peak stress remains the same [2]. The shape of the stress distribution is determined by, among others, the SCF, so using S-N data with a different SCF value than the case at hand may lead to a wrong life estimation. The reference stress amplitude, therefore, needs another translation to be suitable for comparison to the $\mathrm{S}-\mathrm{N}$ data used. In HSB 62131-01 [17], a method for the prediction of the fatigue life for cases with SCF values different to available S-N data is described. The method makes a distinction between two translation methods which are termed 'method 1,' and 'method 2' for the remainder of this paper

If one S-N curve is used, SCF translation method 1 is applied, which entails multiplying the stress amplitude with a load factor $f$ to account for different SCF values of the $\mathrm{S}-\mathrm{N}$ curve. In general, the closer the SCF values, the more accurate the obtained prediction will be. The load factors are calculated using:

$$
f= \begin{cases}K_{t} & K_{t, \mathrm{~S}-\mathrm{N}}=1, K_{t} \geq 1 \\ \frac{K_{t}}{K_{t, \mathrm{~S}-\mathrm{N}}} & K_{t, \mathrm{~S}-\mathrm{N}}>1, K_{t}>K_{t, \mathrm{~S}-\mathrm{N}} \\ 1 & K_{t, \mathrm{~S}-\mathrm{N}}>1, K_{t} \leq K_{t, \mathrm{~S}-\mathrm{N}}\end{cases}
$$

and thus the corrected stress amplitude is found by:

$$
S_{a, \text { corrected }}=f \cdot S_{a, \mathrm{~S}-\mathrm{N}}
$$

which is used to predict the cycles to crack initiation from the corresponding S-N curve.

If more than one $\mathrm{S}-\mathrm{N}$ curve is available, $\mathrm{SCF}$ correction method 2 can be used, where two S-N curves with the same stress ratio are selected to perform an interpolation or extrapolation, depending on the choice of curves, to predict the cycles to crack initiation. A prediction for the cycles to crack initiation is made using each chosen S-N curve, making use of the stress amplitude $S_{a, \mathrm{~S}-\mathrm{N}}$ from section 2.3.2, after which the final result is found using:

$$
\begin{aligned}
& \log (N)=\log \left(N_{2}\right)+ \\
& \quad\left(\log \left(N_{2}\right)-\log \left(N_{1}\right)\right) \frac{\log \left(K_{t}\right)-\log \left(K_{t, \mathrm{~S}-\mathrm{N} 2}\right)}{\log \left(K_{t, \mathrm{~S}-\mathrm{N} 2}\right)-\log \left(K_{t, \mathrm{~S}-\mathrm{N} 1}\right)}
\end{aligned}
$$


Table 1. Crack initiation life test data and corresponding calculated nominal stress amplitude in the metal layers, peak stress ratio and maximum SCF for Glare $4 \mathrm{~B}-3 / 2-0.3$ with $K_{t, \text { iso }}=2.7, R=0.05[7]$

\begin{tabular}{ccccc}
\hline $\begin{array}{c}\boldsymbol{S}_{\boldsymbol{a}, \text { net }} \\
{[\mathbf{M P a}]}\end{array}$ & $\begin{array}{c}\boldsymbol{N}_{\boldsymbol{i}} \\
{[\text { cycles] }}\end{array}$ & $\begin{array}{c}\boldsymbol{S}_{\boldsymbol{a}, \text { nom }} \\
{[\mathbf{M P a}]}\end{array}$ & $\begin{array}{c}\boldsymbol{R}_{\text {peak }} \\
{[-]}\end{array}$ & $\begin{array}{c}\boldsymbol{K}_{\boldsymbol{t}, \text { max }} \\
{[-]}\end{array}$ \\
\hline 110 & 2599 & 160.4 & 0.073 & 2.879 \\
100 & 3993 & 145.8 & 0.075 & 2.866 \\
90 & 6118 & 131.2 & 0.078 & 2.850 \\
80 & 9739 & 116.6 & 0.082 & 2.831 \\
70 & 18427 & 102.1 & 0.086 & 2.807 \\
60 & 34706 & 87.5 & 0.092 & 2.775 \\
50 & 68583 & 72.9 & 0.099 & 2.733 \\
\hline
\end{tabular}

where $N$ is the resulting amount of cycles to initiation, $N_{1}$ and $N_{2}$ are the cycles to initiation using the individual SN curves, and $K_{t, \mathrm{~S}-\mathrm{N} 1}$ and $K_{t, \mathrm{~S}-\mathrm{N} 2}$ the SCF values of the corresponding S-N curves used. The index 1 always points to the S-N curve with the lower SCF value of the two.

The applicability of both the SCF correction methods mentioned above is limited to high cycle fatigue $(N>$ $5 \cdot 10^{3}$ cycles) $[17]$.

\section{Results and discussion}

Homan and Schra [7] have tested the cycles to crack initiation on Glare 4B laminates. The results of one test series on Glare 4B-3/2-0.3 can be found in table 1 . This material has a stiffness of $48.7[\mathrm{GPa}]$ and a nominal tensile yield and ultimate strength of $251[\mathrm{MPa}]$ and $572[\mathrm{MPa}]$ in longitudinal direction [1]. The constituent mechanical properties are given in table 2. More information about (this type of) Glare can be found in [1]. The cycles to initiation were obtained as a function of net section stress amplitude $S_{a \text {, net }}$, which is explained in section 2.2.1. The prediction methodology for cycles to crack initiation described in section 2 is used in this section to predict the cycles to initiation in the same situations. The material properties that were used in the simulation for the Glare constituents were those given by Homan [6] which can be found in 2. Comparing the predictions with the experimental $\mathrm{S}-\mathrm{N}_{\mathrm{i}}$ data obtained by the tests enables conclusions to be made about the validity of the prediction methodology.

First, the influence of the changes the method described by Homan [6] to the method explained in section 2 is assessed by calculating the layer stresses using both methods and looking at the relative difference between both answers. Second, the metal layer stress is calculated for the data in table 1 and subsequently corrected for the $R$-ratio and the $K_{t}$-value of several S-N curves for monolithic aluminium to see how both agree. Third, the influence of the choice of S-N curve and the choice of $K_{t}$ correction method on the prediction accuracy is evaluated on the basis of the difference between prediction and test result. Finally, the method is applied to other FMLs to evaluate its applicability.

\subsection{Layer stress calculation}

Example calculations of the residual stresses and the nominal layer stresses in the aluminium layers for three different Glare laminates are made using both Homan's and the current method. The relative difference between the two is calculated using:

$$
\delta=\frac{S_{\text {Homan }}-S_{\text {current }}}{S_{\text {current }}} \cdot 100 \%
$$

and the result is summarised in table 3 . There is no difference at a $0^{\circ}$ and $90^{\circ}$ load direction. A significant error of up to $60 \%$, however, is observed at a $45^{\circ}$ loading direction for Glare 4B-3/2-0.3. For other laminates and loading directions (other than $0^{\circ}$ and $90^{\circ}$ ) the amount of difference is smaller, although still significant.

\subsection{Comparison to $S-N$ curves from literature}

The aim of this section is to evaluate the accuracy of calculating the reference stress from the far-field load value using the method of section 2 . The resulting data pairs of reference stress amplitude and cycles to initiation are compared with legacy S-N data for aluminium 2024-T3. A good agreement between the two means that a prediction for FCI in Glare can indeed be made using S-N curves for monolithic aluminium available in literature.

The $\mathrm{S}-\mathrm{N}_{\mathrm{i}}$-data for notched Glare 4B-3/2-0.3 specimens with $K_{t}=2.7$ [7] is compared to S-N data for monolithic aluminium 2024-T3 obtained from HSB [18], see figures 5, 6,7 , and 8 . The value of the SCF is what it would be if isotropic specimens of the same geometry would be used.

Three different sets of data points can be seen in each figure, along with an S-N curve. The empty circles correspond to the average far-field laminate stress that was applied in the tests. The solid squares are the corresponding nominal stress values in the metal layers of the FML, and the solid triangle are the results of adapting the nominal stress value to the $K_{t}$ and $R$ value of the S-N curve the data is compared to, according to section 2.3.

\subsubsection{Metal layer stress instead of applied stress}

Glare withstands a smaller net section stress than monolithic aluminium to reach comparable crack initiation lives, compare the circles in e.g. figure 5 with the line. Homan already indicated that the stresses occurring in the aluminium layers should be used to compare FMLs to monolithic materials [6]. The data points then lie much closer to the S-N curve, as can be seen by the squares in the same figure. 
Table 2. Mechanical and physical properties of Glare constituents [6] that served as input for the prediction methodology

\begin{tabular}{cccc}
\hline Material properties & & Aluminium 2024-T3 & S2-glass $/$ FM94 epoxy \\
\hline Youngs modulus [MPa] & $E_{1}$ & 72,400 & 48,900 \\
Shear modulus [MPa] & $E_{2}$ & 72,400 & 5,500 \\
Poissons ratio [-] & $\nu_{12}$ & 26,900 & 5,550 \\
& $\nu_{21}$ & 0.33 & 0.33 \\
Coefficient of & 0,33 & 0,0371 \\
thermal expansion $\left[1 /{ }^{\circ} \mathrm{C}\right]$ & $\alpha_{1}$ & $22 \cdot 10^{-6}$ & $6.1 \cdot 10^{-6}$ \\
Layer thickness $[\mathrm{mm}]$ & $\alpha_{2}$ & $22 \cdot 10^{-6}$ & $26.2 \cdot 10^{-6}$ \\
Cure temperature [ $\left.{ }^{\circ} \mathrm{C}\right]$ & $T$ & 0.3 & 0.133 \\
\hline
\end{tabular}

Table 3. Percentile difference between Homan's [6] and the current method to calculate residual stress and total layer stress for several Glare types and loading angles at an applied stress of 70 [MPa], calculated using equation (25).

\begin{tabular}{ccccccc}
\hline Glare & \multicolumn{3}{c}{ Residual stress } & \multicolumn{3}{c}{ Layer stress* } \\
type & $\mathbf{0}^{\circ}$ & $\mathbf{2 2 . 5}^{\circ}$ & $\mathbf{4 5}^{\circ}$ & $\mathbf{0}^{\circ}$ & $\mathbf{2 2 . 5}^{\circ}$ & $\mathbf{4 5}^{\circ}$ \\
\hline $2 \mathrm{~A}-3 / 2-0.3$ & 0 & -19.3 & -35.6 & -3.4 & -5.8 & -6.1 \\
$3-3 / 2-0.5$ & 0 & -6.1 & 33.9 & -3.6 & -3.1 & -1.1 \\
$4 \mathrm{~B}-3 / 2-0.3$ & 0 & 26.8 & 60.0 & -3.6 & -0.7 & 4.0 \\
\hline
\end{tabular}

*The layer stress includes the contribution of applied load and residual stress

\subsubsection{Applying corrections to approach the $S$ - $N$ data}

The translation explained above would be very accurate if the $K_{t, \mathrm{~S}-\mathrm{N}}$ and $R_{\mathrm{S}-\mathrm{N}}$ of the $\mathrm{S}-\mathrm{N}$ curve are equal to the $K_{t}$ and $R$ occurring in the aluminium layers of the test case. This is, however, rarely the case, and additional translations are necessary to obtain a good agreement between the test data and S-N curves. In sections 2.3.2 and 2.3.3, corrections were introduced to consider the differences between the $K_{t}$ and $R$ values of test case and S-N data. To find out the range of applicability of both correction methods, an analysis is performed using aluminium $\mathrm{S}$ $\mathrm{N}$ data with a variety of $K_{t, \mathrm{~S}-\mathrm{N}}$ and $R_{\mathrm{S}-\mathrm{N}}$ values, obtained from HSB [18]. Method 1 has been chosen to correct for a difference in SCF in this section.

As can be concluded from figures 5 to 8, the correction results in a very good agreement for the cases where the value of $K_{t}$ in the S-N data lies below that of the predicted case, as shown in figures 5 and 6 . The method is far less accurate for the other two cases.

When $K_{t}$, S-N is larger than $K_{t, \max }$, applying the correction $f=\frac{K_{t, \max }}{K_{t, \mathrm{~S}-\mathrm{N}}}$ would result in an unconservative prediction, because the points would fall significantly below the S-N curve. Equation (22), therefore, is such that it does not adapt stress amplitude in case the $K_{t, \mathrm{~S}-\mathrm{N}}$ falls below $K_{t \text {, max }}$ and thus the original amplitude is used to obtain a conservative prediction [17].

It seems from the data considered that when the test data is compared to S-N curves with a $K_{t}$, S-N value lower

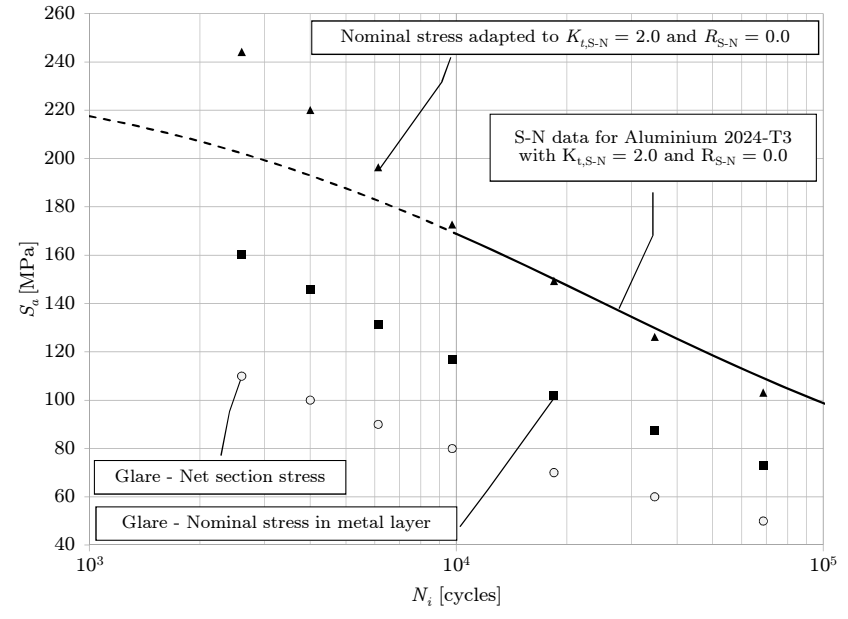

Figure 5. Comparison of crack initiation life measurements of Glare $4 \mathrm{~B}-3 / 2-0.3[7]$ to an S-N curve with $K_{t}=2.0$ and $R=0$, valid above $N=10^{4}$ cycles[18].

than the $K_{t, \max }$, a good agreement is obtained when both corrections to the stress amplitude are applied. When the $K_{t, \mathrm{~S}-\mathrm{N}}$ of the used S-N curve is higher than $K_{t, \max }$, the agreement is not so good but conservative, because only the stress ratio is corrected. The case with $K_{t, \mathrm{~S}-\mathrm{N}}=1$ was analysed as well. Unfortunately, no match was obtained and all estimations gave results higher than the run-out of $10^{7}$.

The same analysis was performed for S-N curves with $R=0.25, R=-0.5$ and $R=-1$, where similar results were obtained: the correction for the stress ratio always improves the agreement and the correction for the stress concentration factor only if the $K_{t, \mathrm{~S}-\mathrm{N}}$ is smaller than $K_{t, \max }$ and not equal to 1 . Applying the corrections according to this case resulted in a good agreement for all cases analysed.

The S-N curves are only valid for load levels that result in $10^{4}$ to $10^{7}$ cycles to crack initiation [18]. The method described in this paper therefore cannot be verified by comparing the Glare test results with higher load levels than these data. Comparing the trend of the corrected test data with the S-N curve in figure 6 , however, does allow for a conclusion to be made about these higher loads. S-N 


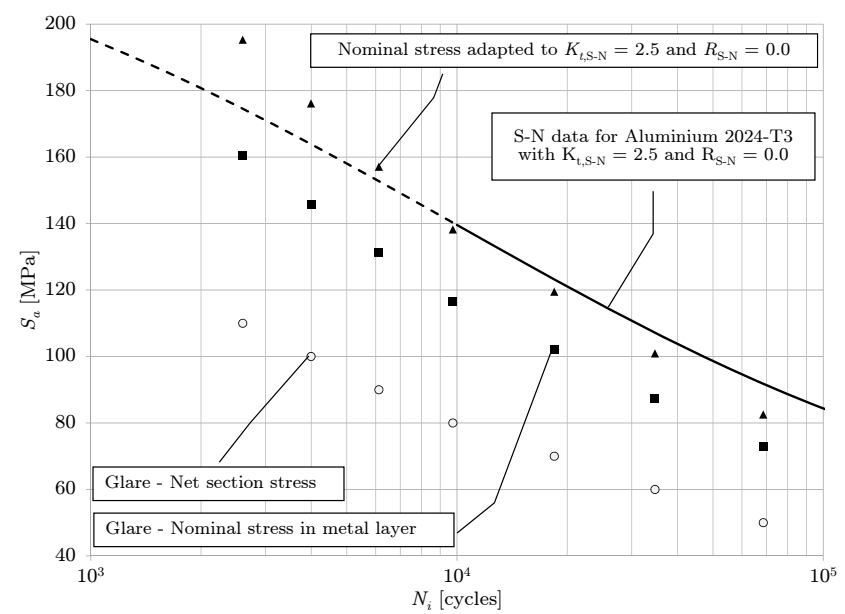

Figure 6. Comparison of crack initiation life measurements of Glare 4B-3/2-0.3 [7] to an S-N curve with $K_{t}=2.5$ and $R=0$, valid above $N=10^{4}$ [cycles] [18].

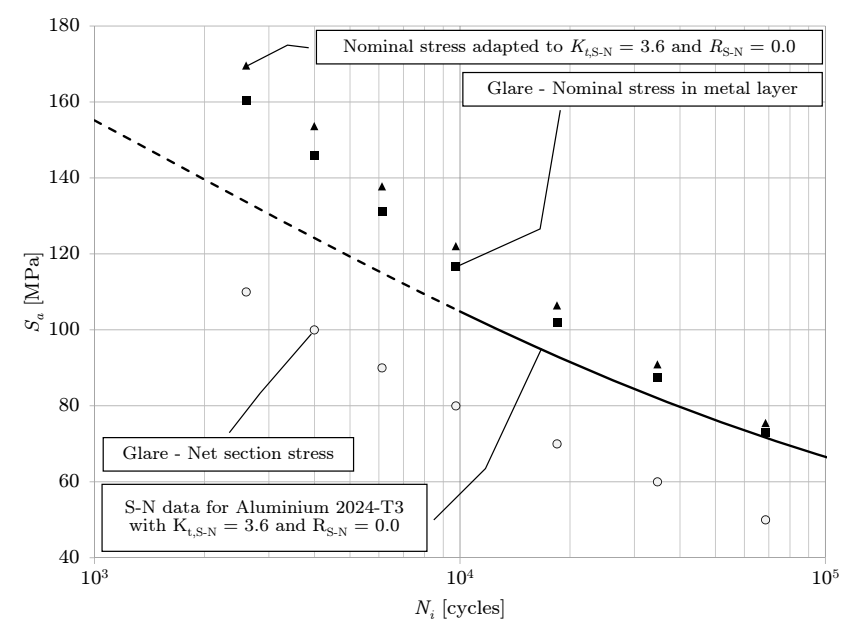

Figure 7. Comparison of crack initiation life measurements of Glare 4B-3/2-0.3 [7] to an S-N curve with $K_{t}=3.6$ and $R=0$, valid above $N=10^{4}$ [cycles] [18].

curves show a decreasing slope towards a maximum stress close to the ultimate stress in the direction of the stressaxis [2]. This means that corrected data points with stress levels above $140[\mathrm{MPa}]$ in figure 6 will fall above the $\mathrm{S}-\mathrm{N}$ curve. This leads to conservative predictions at load levels above the maximum load level of the S-N data, because the proposed method will predict a lower amount of cycles if S-N data were available at these levels.

\subsection{Evaluation of FCI predictions}

The predicted cycles to initiation using S-N curves of monolithic notched metal plates are compared to $\mathrm{S}-\mathrm{N}_{\mathrm{i}}$-test results of notched FML plates in this section.

The accuracy of the predictions is evaluated using the absolute value of the ratio of the predicted cycles to crack initiation with the expected value, i.e. the test result, as

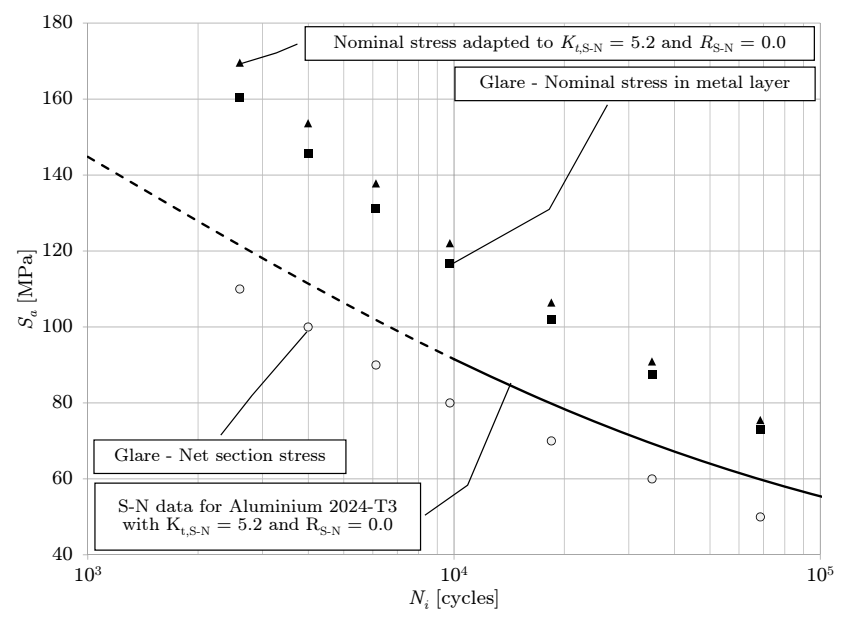

Figure 8. Comparison of crack initiation life measurements of Glare 4B-3/2-0.3 [7] to an S-N curve with $K_{t}=5.2$ and $R=0$ [18].

Table 4. Average error $\eta$, see equation (26), of fatigue crack initiation life predictions for Glare $4 \mathrm{~B}-3 / 2-0.3$ with $K_{t}$, iso $=2.7, R=0.05[7]$ to replicate the test results of table 1.

\begin{tabular}{ccccc}
\hline & \multicolumn{4}{c}{$\boldsymbol{R}_{\mathrm{S}-\mathrm{N}}$} \\
\cline { 2 - 5 } $\boldsymbol{K}_{\boldsymbol{t}, \text { S-N }}$ & $\mathbf{0 . 2 5}$ & $\mathbf{0 . 0}$ & $\mathbf{- 0 . 5}$ & $\mathbf{- 1 . 0}$ \\
\hline $\mathbf{2 . 0}$ & $0.27^{2}$ & $0.19^{2}$ & $0.29^{2}$ & $0.64^{2}$ \\
$\mathbf{2 . 5}$ & 0.45 & 0.33 & $0.49^{1}$ & $0.88^{2}$ \\
$\mathbf{3 . 6}$ & $0.51^{1}$ & $0.51^{1}$ & $0.41^{2}$ & $0.46^{2}$ \\
$\mathbf{5 . 2}$ & $0.67^{1}$ & $0.72^{2}$ & $0.71^{3}$ & $0.71^{3}$ \\
\hline $\mathbf{2 . 0}$ \& $\mathbf{2 . 5}$ & 0.76 & 0.38 & $0.54^{1}$ & $0.76^{1}$ \\
$\mathbf{2 . 0} \& \mathbf{3 . 6}$ & $0.30^{1}$ & $0.31^{1}$ & $0.57^{2}$ & $1.60^{2}$ \\
$\mathbf{2 . 0} \& \mathbf{5 . 2}$ & $0.95^{1}$ & $0.66^{2}$ & $1.10^{3}$ & $2.87^{3}$ \\
$\mathbf{2 . 5}$ \& $\mathbf{3 . 6}$ & $0.53^{1}$ & $0.34^{1}$ & $0.57^{2}$ & $1.07^{2}$ \\
$\mathbf{2 . 5}$ \& $\mathbf{5 . 2}$ & $0.90^{1}$ & $0.52^{2}$ & $0.71^{3}$ & $1.34^{3}$ \\
$\mathbf{3 . 6}$ \& $\mathbf{5 . 2}$ & $0.36^{1}$ & $0.30^{2}$ & $0.38^{3}$ & $0.69^{3}$ \\
\hline Excluding ${ }^{3} 110{ }^{2} 100$ and 110, and $^{3} 90,100$, and $110[\mathrm{MPa}]$
\end{tabular}

a measure of relative error:

$$
\eta=\left|\frac{N_{\text {predicted }}}{N_{\text {expected }}}-1\right|
$$

Table 4 contains the average error of the prediction of multiple data points for the FCI in notched Glare 4B-3/20.3 using various $\mathrm{S}-\mathrm{N}$ curves for notched aluminium. A value in the table belongs to predictions that are made using the S-N curve(s) of the $R$ - and $K_{t}$-values indicated. These values show the average error to predict the cycles to initiation for each data point in table 1 , which is determined by using equation (26) for each data point and taking the average value of the set of results. When two values for $K_{t}$ are indicated in table 4 , method 2 was applied, otherwise method 1 was used.

S-N curves with $K_{t}=1$ give only run-outs for the tested laminate and are thus excluded from the comparison in table 4 . The various load levels that were also excluded from a large part of the table entries are a result 
of the method failing to produce an answer when the stress amplitude lies above the highest value of the S-N curve.

The data in table 4 shows that the accuracy of the prediction methodology is strongly influenced by the choice of $\mathrm{S}-\mathrm{N}$ data that is to serve as the base of the predictions. It is important to realise that a bad agreement of a prediction with test results, therefore, does not necessarily disprove the validity of the method. It can simply mean that the S-N data does not represent the metal layers in the FML to a satisfactorily degree. The opposite is also true: a good agreement between predictions and test result does not validate the method, but could be a lucky choice of $\mathrm{S}-\mathrm{N}$ data. It is thus important to test the methodology in a large variety of cases to establish its validity.

\subsubsection{Accuracy of the predictions}

The average error over all rows equals $0.57,0.43,0.58$, and 1.10 for $\mathrm{S}-\mathrm{N}$ data with $R=0.25,0.0,-0.5$, and -1.0 , respectively. The applied load cycle has $R=0.05$, leading to $0.073 \leq R_{\text {peak }} \leq 0.099$. The $\mathrm{S}-\mathrm{N}$ curves with the closest value for the load ratio have $R=0.0$, and the average error of the column with this value for $R$ is also the lowest of the four. This leads to the conclusion that indeed the best predictions are obtained when the load ratio of the $\mathrm{S}-\mathrm{N}$ data equals the case analysed.

The case analysed has $K_{t, \max }=2.807$, so in this case the S-N curve with $K_{t, \mathrm{~S}-\mathrm{N}}=2.5$ lies closest to this value. It can be seen in table 4 that the error using a single S-N curve with this value of $K_{t}$ is not the smallest in comparison to using other curves to predict crack initiation. This is probably a result of scatter of the test data that is used to evaluate the accuracy, and could be solved using a larger dataset.

Based on the trends in the average error values using method 1, it seems that it is beneficial to use S-N data that has a lower $K_{t}$ value than the case for which a prediction is made, rather than a higher one. This is probably a result of equation (22), which only corrects the prediction in case the SCF of the S-N curve falls below the case analysed, as mentioned in section 3.2.2.

\subsubsection{Method 1 versus method 2}

It was mentioned earlier that the column with the most accurate results is $R=0.0$. The average error of the predictions in this column using method 1 equals 0.44 . The average error is 0.42 when method 2 is applied. It thus appears that both methods produce predictions that seem equally accurate on average and no decision can be made on whether one method is significantly better than the other. A slight preference of method 1 over method 2 could be based on the high errors seen using the latter method when the $R$ value is far off from the case analysed, while method 1 remains relatively accurate.

The result of the two prediction methods is plotted with the test results for a variety of stress amplitudes in figure 9 . The error, as defined by equation (26), is plotted per stress level in figure 10. For each data point, the S-N

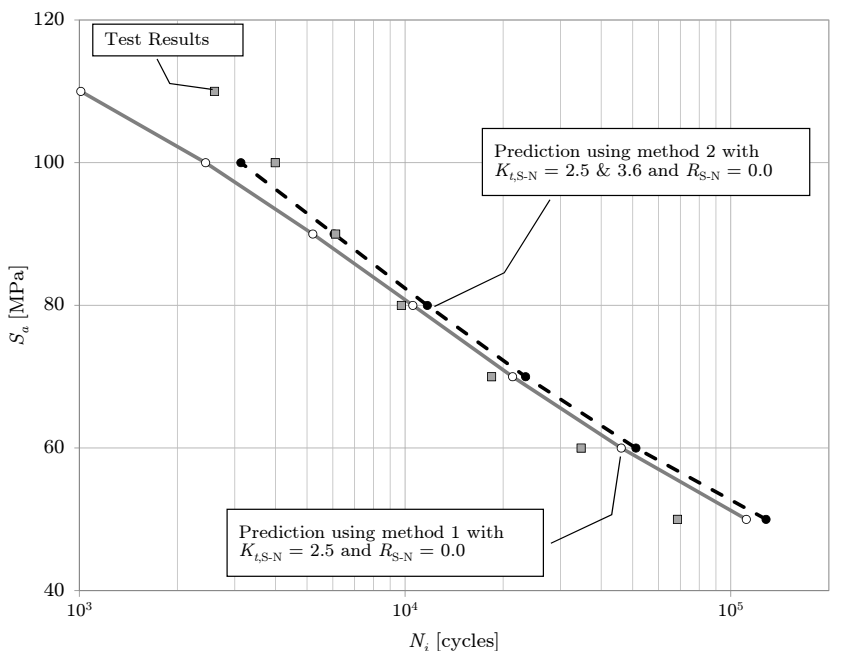

Figure 9. Fatigue crack initiation life predictions for Glare 4B-3/20.3 at several stress amplitudes using both methods mentioned in section 2.3.3 compared to test results from table 1 .

curves were used that had the smallest difference in $K_{t}$ and $R$ values with the case analysed: $K_{t, \mathrm{~S}-\mathrm{N}}=2.5$ for method 1 , and $K_{t, \mathrm{~S}-\mathrm{N}}=2.5$ and 3.6 for method 2 , and $R=0$ for all S-N data used.

It can be seen in the figures that both prediction methods approach the test results quite well. The amount of error is low at stress amplitudes in the middle of the range tested, and increases when the applied stress amplitude either decreases or increases. The increasing error towards a higher applied load is a result of the fact that the S-N data used is not valid below $10^{4}$ cycles [18], but it is still used to make a prediction, see also the dashed part of the $\mathrm{S}-\mathrm{N}$ curves in figures 5 to 8 . The increasing error towards a lower applied load is a result of the decreasing slope of the $\mathrm{S}-\mathrm{N}$ curves towards the fatigue limit. This causes a larger variation in cycles to initiation as compared to a higher load, for the same variation in stress amplitude.

There is no data point for method 2 at $S_{a}=110$ [MPa], because the method predicts failure below a single cycle as a result of trying to find a value above the upper stress limit of the curve that constitutes the S-N data [18]. It is decided not to include any result outside the limits of the curve, which lie at $10^{0}$ and $10^{7}$ cycles.

\subsubsection{Influence of stress concentration factor}

The SCF is not constant during the application of a load cycle, as was mentioned in section 2.2. A $K_{t, \max }$ and a $K_{t}$, min are calculated for each occurring stress cycle, based on the maximum and minimum peak stress.

The prediction of the cycles to crack initiation for Glare 4B-3/2-0.3 with $S_{a}=70[\mathrm{MPa}]$ is plotted in figure 11 . The $K_{t}$ value is varied between $K_{t}$, min and $K_{t, \max }$, as is indicated by the vertical dotted lines in the figure. The solid grey line belongs to the result of using a single S-N curve each time, and the dashed black line to the result of interpolating between two S-N curves. The dash-dot 


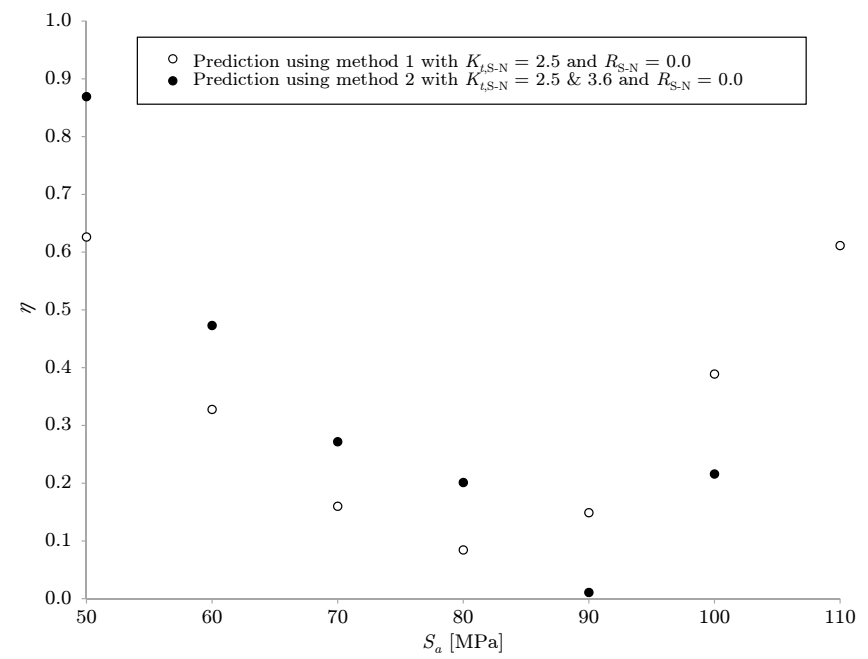

Figure 10. Error $\eta$ of the crack initiation predictions for Glare 4B$3 / 2-0.3$ in figure 9 compared to test results in table 1 , calculated using equation (26).

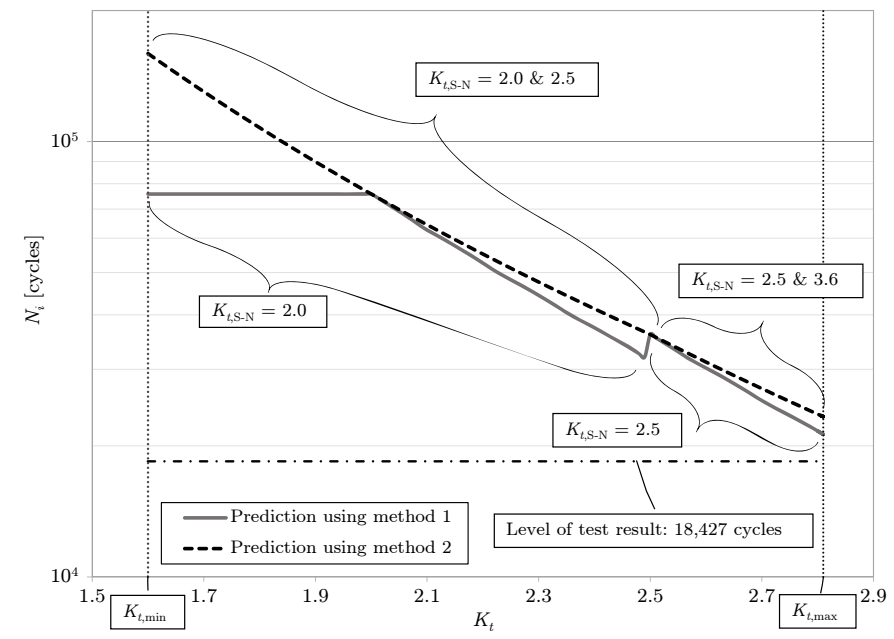

Figure 11. Prediction for the cycles to crack initiation of Glare 4B$3 / 2-0.3$ at $S_{a}$, net $=70[\mathrm{MPa}]$ using a SCF value between $K_{t, \text { min }}=$ 1.60 and $K_{t, \max }=2.81$ and $R_{\mathrm{S}-\mathrm{N}}=0$.

line in the figure represents the number cycles to initiation determined from fatigue testing.

The S-N curve with the value of $K_{t, \mathrm{~S}-\mathrm{N}}$ closest but below the analysed SCF value was chosen each time for method 1 , because equation (22) does not correct for cases where $K_{t} \leq K_{t, \mathrm{~S}-\mathrm{N}}$. The S-N curves which form the limits of an interval of available $K_{t}$, S-N values, or the closest two in case $K_{t}$ lies below 2.0 or above 5.2 , were chosen for method 2 .

The figure clearly shows that, regardless of the SCF correction method, using the $K_{t}$, max results in an answer that lies closest to the measured value. This is an expected result, since the maximum peak stress is the most severe stress occurring and thus it is likely to drive damage initiation. Repeating this analysis for other load cycles consistently leads to the same conclusion.

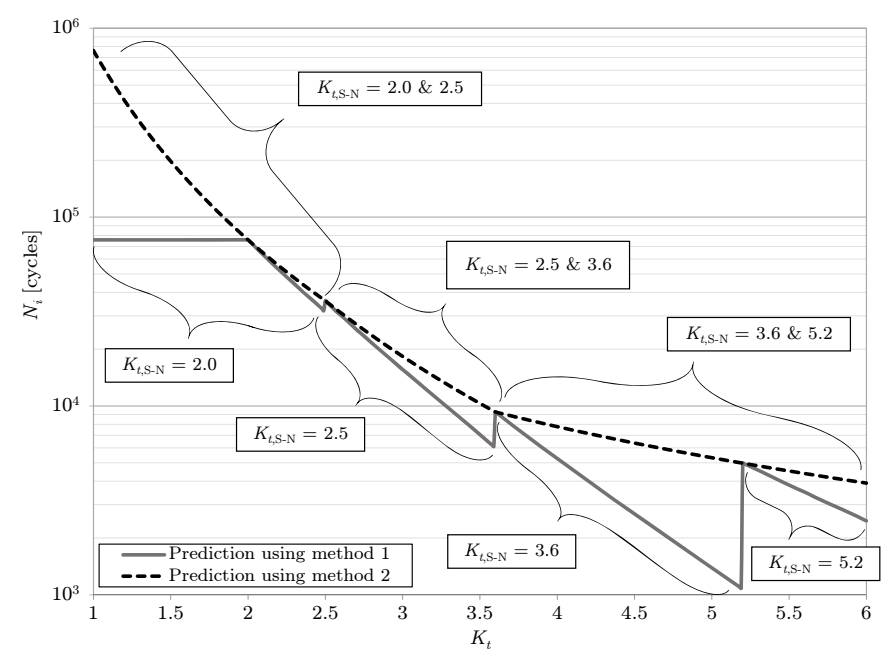

Figure 12. Prediction based on $S_{a, \text { nom }}=102.1$ [MPa], $R_{\text {peak }}=$ 0.859 varied between $K_{t}=1.0$ and $K_{t}=6.0$ using the closest available S-N data with $R_{\mathrm{S}-\mathrm{N}}=0$.

\subsubsection{Sensitivity of estimated fatigue life}

Two methods were explained above to correct the SCF for the estimation of the cycles to initiation. In figure 12, the results of both estimation methods are compared for a larger range of $K_{t}$ values than in figure 11 .

The sudden steps or slope changes in the results clearly show the switch from using one (set of) S-N curve(s) to another. Method 1, moreover, keeps giving the same life estimation if the $K_{t}$ value of the closest S-N curve is higher than the occurring $K_{t}$ value, as can be seen by the horizontal portion of the solid grey line in the figure.

Method 2 shows a smoother transition from interpolating using one set of curves to another. This behaviour is preferred especially in case of automated optimisation of FML layups for crack initiation, because it means the optimiser will see a gradient in the results which it can use to find an optimum. No horizontal sections can be seen in the dashed line, indicating that the estimation below the lowest $\mathrm{S}-\mathrm{N}$ curve is probably more accurate using interpolation. This was already concluded from the lower observed error in section 3.3.1.

\subsubsection{Other materials}

The presented prediction methodology proved to give acceptable predictions for Glare 4B-3/2-0.3. In order to show that the method is generic and can cope with any type of FML, the validation process is extended to different laminates. In total, four additional laminates were investigated from which the cycles to initiation life is predicted and compared to test results found in the literature.

Bradshaw and Gutierrez [19] present crack initiation test data for aluminium 2024-T3 sheets combined with a carbon/epoxy laminate. The FML has a layup that can be written as $[\mathrm{Al} / 45 / 0 /-45 / 90 / 45 / 0 /-45]_{s}$, where $\mathrm{Al}$ stands for a $0.203[\mathrm{~mm}]$ thick sheet. The type of the fibre ply in the laminate is not specified in the paper. Neverthe- 


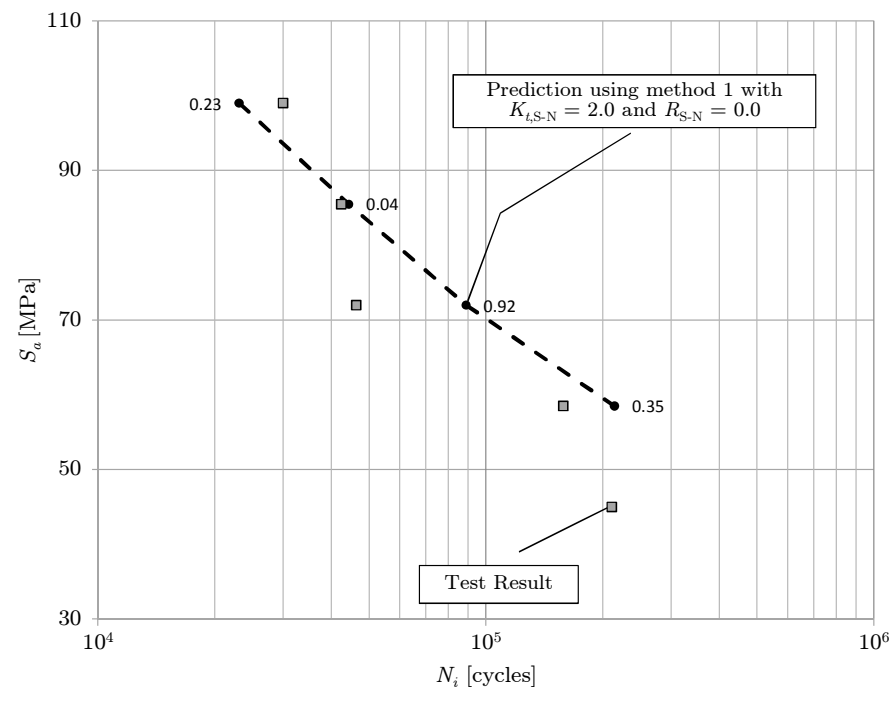

Figure 13. Fatigue crack initiation life predictions for an aluminium carbon/epoxy laminate with a $[\mathrm{Al} / 45 / 0 /-45 / 90 / 45 / 0 /-45]_{s}$ layup at several stress amplitudes using method 1 compared to test results from [19].

less, Alderliesten and Rans [20] determined with the use of reverse engineering that the prepreg layers consist of T800 carbon fibre plies with a $0.188[\mathrm{~mm}]$ thickness. Multiple specimens were tested for each load case and the given test results in figure 13 are the mean values at each stress amplitude. The test data of this laminate is compared to the predicted results obtained using method 1 and an $\mathrm{S}-\mathrm{N}$ curve for aluminium with $K_{t, \mathrm{~S}-\mathrm{N}}=2.0$ and $R_{\mathrm{S}-\mathrm{N}}=0.0$ [18]. The relative error with respect to the test data is given next to each test data point. The predictions show a good agreement with the test data. More information on the material properties and test set-up of the test data can be found in [19].

Chang et al. [9] have tested aluminium 2024-T3 laminates with $\mathrm{S} 2$-glass fibre and boron fibre layers in the layup $[\mathrm{Al} / 0, \mathrm{~S} 2 / 0, \mathrm{~B} / 0, \mathrm{~S} 2 / \mathrm{Al}]$, where $\mathrm{Al}$ indicates a 0.3 [mm] aluminium layer, 0, S2 a 0.133 [mm] S2-glass layer with fibres in longitudinal specimen direction, and $0, \mathrm{~B}$ a 0.133 [mm] boron fibre layer, again with fibres in longitudinal specimen direction. In figure 14, the comparison of the data points are given with a prediction using an $\mathrm{S}-\mathrm{N}$ curve for aluminium with $K_{t, \mathrm{~S}-\mathrm{N}}=2.0$ and $R_{\mathrm{S}-\mathrm{N}}=0.0$ [18]. It can be observed that the predictions are acceptable for high stress levels, but the predictions tend to be too optimistic for the lower stress levels. All in all the agreement is reasonable.

Homan $[6,7]$ performed crack initiation tests on Glare 3-3/2-0.3 laminates as well, next to the tests on Glare 4B$3 / 2-0.3$ that were thoroughly treated earlier. The result for this Glare laminate is given in figure 15. An S-N curve with $K_{t, \mathrm{~S}-\mathrm{N}}=2.5$ and $R_{\mathrm{S}-\mathrm{N}}=0.25$ [18] was used to make predictions. The prediction at lower stress levels agree well with the test data, while at higher stresses the prediction tends to become too conservative. For the highest two stresses no predictions were obtained since these were out-

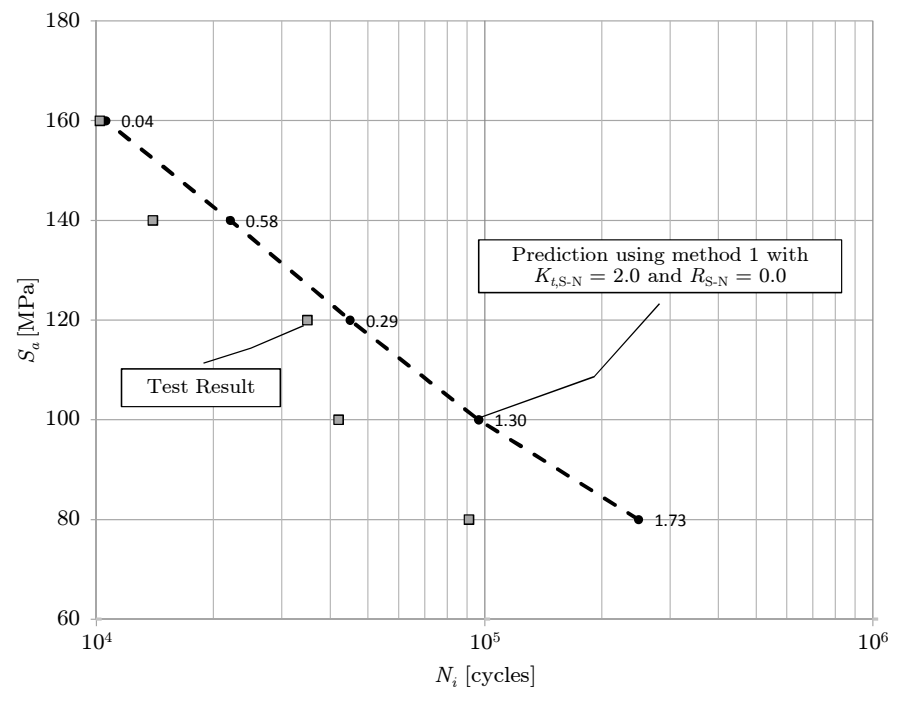

Figure 14. Fatigue crack initiation life predictions for a $[\mathrm{Al} / 0, \mathrm{~S} 2 / 0, \mathrm{~B} / 0, \mathrm{~S} 2 / \mathrm{Al}]$ aluminium boron laminate at several stress amplitudes using method 1 compared to test results from [9].

of-the-range of the S-N curve.

Finally, predictions are made for a stainless steel laminate. Shahinian [21] investigated carbon fibre-reinforced stainless steel laminates with the following configuration: $[\mathrm{St} / \mathrm{C} / \mathrm{St} / \mathrm{C} / \mathrm{St}]$. Here St stands for a $5.0[\mathrm{~mm}] 316 \mathrm{~L}$ steel layer and $\mathrm{C}$ for a 0.13 [mm] T700/FM94 carbon/epoxy layer. In figure 16, the test results are given, along with predictions. The S-N data for steel is obtained from [21] as well, in which monolithic 316L steel specimens with a thickness of $5.0[\mathrm{~mm}]$ were tested at different stress levels with $K_{t, \mathrm{~S}-\mathrm{N}}=2.7$ and $R_{\mathrm{S}-\mathrm{N}}=0.05$. The predictions are all close to the test data, albeit on the optimistic side.

\section{Conclusions}

A complete methodology to obtain a prediction for cycles to crack initiation in an FML has been presented. Due to the presence of thermal stress, $K_{t}$ was found not to remain a purely geometrical factor, but to vary with the amount of load that is applied to the specimen along the load cycle. The stress ratio in a region of stress concentration shows the same load-dependent behaviour in the presence of thermal stress. Using the values for $K_{t}$ and $R$ at maximum applied load to predict the cycles to crack initiation led to the best result.

Two methods were found to use S-N data with a different $K_{t}$ value than the case analysed to predict the cycles to crack initiation: a correction and an interpolation method. Both methods showed a comparable accuracy in predicting the test results that were used to validate the complete methodology. In a optimisation environment, though, the interpolation method has preference over the correction method, because it shows a smoother dependence on the SCF as compared to the correction method.

It was shown that the number of cycles to crack initiation of several types of FML can be predicted accurately 


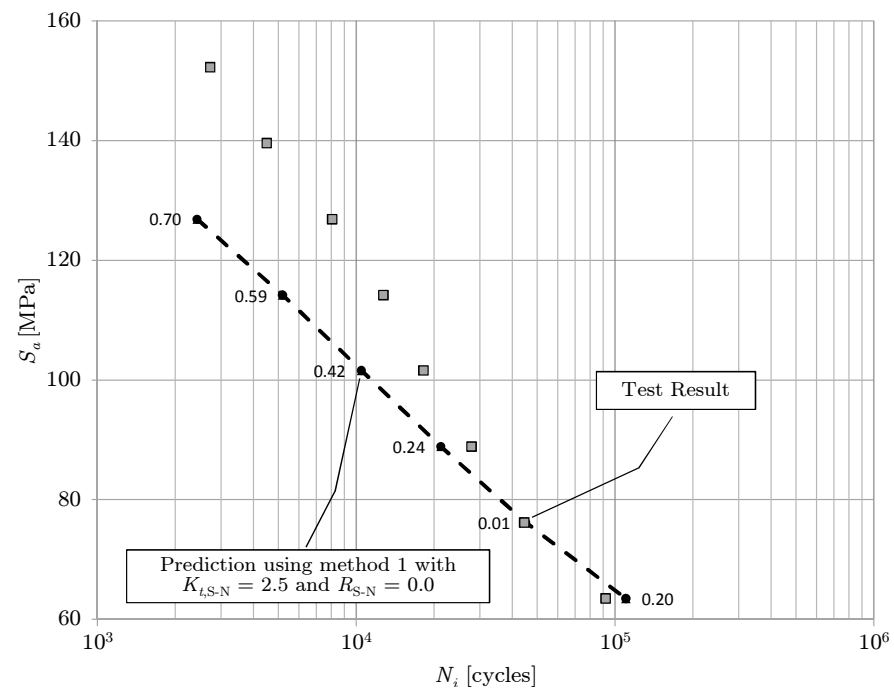

Figure 15. Fatigue crack initiation life predictions for Glare 3-3/2-0.3 at several stress amplitudes using method 1 compared to test results from $[6,7]$.

using the presented methodology. The obtained accuracy depends on the S-N data that is used to make the predictions with. It was shown for Glare $4 \mathrm{~B}-3 / 2-0.3$ that the closer the value of $R$ of the $\mathrm{S}-\mathrm{N}$ data approaches the value of the case analysed, the higher the accuracy obtained. Such a trend was not observed for the $K_{t}$ value, although a choice for S-N data with a different $K_{t}$ can cause a significant change in precision. The method produces accurate results for several other FMLs as well, showing its general applicability.

\section{Acknowledgements}

The authors would like to thank dr. D. Zarouchas and dr. M. Mahdavi-Shahri for their patience in the numerous discussions about this paper, and dr. S. Koussios for sharing his knowledge on the tangential stress around a circular cut-out.

\section{References}

[1] A. Vlot, J. W. Gunnink, Fibre Metal Laminates, an introduction, Kluwer Academic Publishers, Dordrecht, The Netherlands, 2001.

[2] J. Schijve, Fatigue of Structures and Materials, Springer, 2009, ISBN-13: 978-1-4020-6807-2.

[3] A. Vlot, Glare, history of the development of a new aircraft material, Kluwer Academic Publishers, Dordrecht, The Netherlands, 2001.

[4] R. C. Alderliesten, On the available relevant approaches for fatigue crack propagation prediction in glare, International Journal of Fatigue 29 (2007) 289-304.

[5] R. C. Alderliesten, Analytical prediction model for fatigure crack propagation and delamination growth in glare, International Journal of Fatigue 29 (2007) 628-646.

[6] J. J. Homan, Fatigue initiation in fibre metal laminates, International Journal of Fatigue 28 (2006) 366-374.

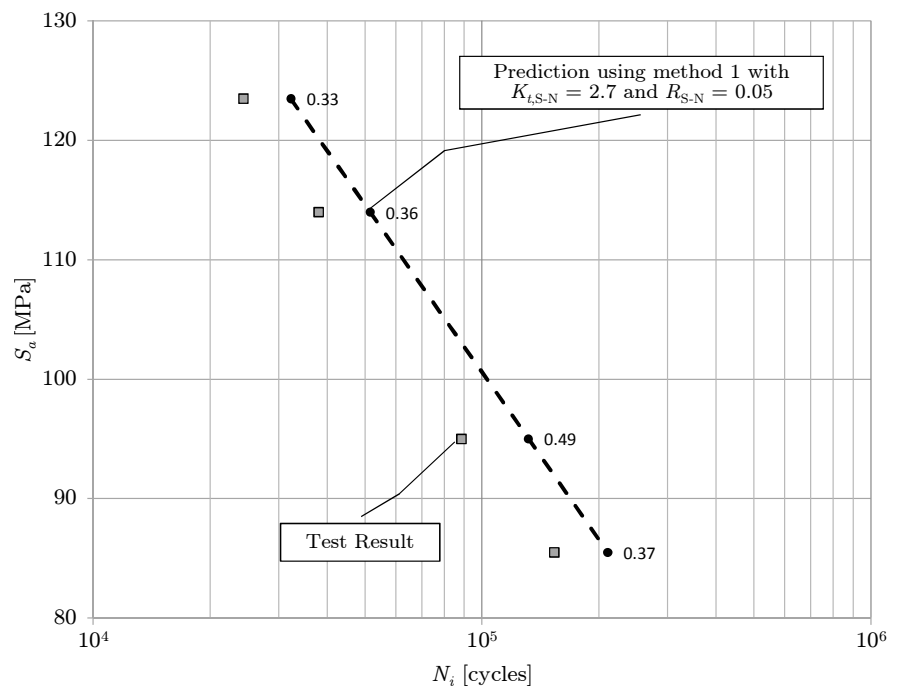

Figure 16. Fatigue crack initiation life predictions for stainless steel carbon/epoxy laminate with a layup of $[\mathrm{St} / \mathrm{C} / \mathrm{St} / \mathrm{C} / \mathrm{St}]$ at several stress amplitudes using method 1 compared to test results from [21].

[7] J. J. Homan, L. Schra, Application of aluminium alloy 2024T3 fatigue life data to Glare laminates, GTP methods projects 2.4.3.2-B, 2.4.3.3-B and 2.3.3.4-B, NLR Report NLR-CR-2002185, National Aerospace Laboratory of the Netherlands (June 2002).

[8] P.-Y. Chang, J.-M. Yang, H. Seo, H. T. Hahn, Off-axis fatigue cracking behaviour in notched fibre metal laminates, Fatigue \& Fracture of Engineering Materials \& Structures 30 (2007) 11581171.

[9] P.-Y. Chang, P.-C. Yeh, J.-M. Yang, Fatigue crack initiation in hybrid boron/glass/aluminum fiber metal laminates, Materials Science and Engineering A 496 (2008) 273-280.

[10] S. Tsai, H. Hahn, Introduction to composite materials, Technomic Publishing Company Inc., 1980.

[11] S. Lekhnitskii, Anisotropic plates, Routledge, reprint edition, 1968.

[12] R. Peterson, Stress Concentration Factors, John Wiley \& Sons Inc., 1974.

[13] Stress concentration factors, Tech. rep., Engineering Sciences Data Unit, London.

[14] R. B. Heywood, Designing against Fatigue, Chapman and Hall, London, 1962.

[15] T. Beumler, Flying Glare, A contribution to aircraft certification issues on strength properties in non-damaged and fatigue damaged Glare structures, PhD dissertation, Delft University of Technology, 2004.

[16] R. Alderliesten, Fatigue and damage tolerance of hybrid materials \& structures - some myths, facts \& fairytales., in: ICAF 2009, ICAF 2009, Bridging the Gap between Theory and Operational Practice, 2009, pp. 1245-1260.

[17] J. J. Homan, Handbuch Struktur Berechnung (HSB), 62131-01 issue A: Guidelines for the prediction of the fatigue life for $\mathrm{Kt}$ values different to available databases, Tech. rep. (2009).

[18] V. Hoang, L. Schwarmann, Handbuch Struktur Berechnung (HSB), 63111-01 issue D: Zeitfestigkeit 3.1354 T3, Tech. rep. (1986).

[19] R. Bradshaw, S. Gutierrez, Characterization of fatigue crack initiation and growth in hybrid aluminium-graphite fibre composite laminates using image analysis, Fatigue and Fracture of Engineering Materials and Structures 30 (2007) 766-781.

[20] R. Alderliesten, C. Rans, If knowing is half the battle, the understanding is the other half: Example of reverse engineering to reconstruct data on boeing laminates, in: $10^{\text {th }}$ Boeing Sponsored Industry Wide Unitized Structures Technical Interchange Conference, 2008. 
[21] R. Shahinian, The next generation Fibre Metal Laminates: A preliminary study of the material properties of carbon fibrereinforced stainless steel laminates, Internship report, Delft University of Technology, 2006. 